\title{
Practical strategies to combat socially transmitted conditions/non-communicable diseases (NCDs)
}

\author{
Suresh Kumar Rathi',*, Mohan Ghule ${ }^{2}$ \\ ${ }^{1,2}$ Self Care Trust
}

*Corresponding Author:

Email: selfcaretrust@gmail.com

The current demographic shift is more likely to be associated with urbanization and health care due to increase in aging population, markedly rise in noncommunicable diseases (NCDs), and rise in terminally ill patients. More so, rapid urbanization has led to increased morbidity and mortality from NCDs (Chronic Diseases). Diabetes and Coronary Artery diseases are two of the Captains of the Battalions of death. Cancer is still probably one of the most dreadful words in English lexicon. People equate cancer with death. The morbidity from cancers, cardiovascular diseases and diabetes in India is alarmingly high ${ }^{1}$ and the high prevalence of NCDs means that more people are developing complications. During last 30-40 years, there is a shift in death place i.e., from hospital to home. It is most likely due to rise of NCDs. We are born with diarrhoea (infectious/ communicable diseases) and will die with diabetes (NCDs). Over the past 10 years, discussions about heart diseases, diabetes and cancers have moved from the doctor's room to the living rooms and club meetings. Cardiac catheterization, angioplasty, and insulin are now part of the vocabulary of people in their 40's and 50's. Coupled with this, limited understanding, services, resources, patients' experiences with managing hypertension and diabetes are some of the issues to be considered while managing NCDs. Nonetheless, we are less prepared and system is less likely to respond and take care of these. In-fact, all these have a significant effect on population and public kitty. Nevertheless, epidemiologists / public health experts already pointed out the NCDs epidemic during early 60's. ${ }^{2}$ In India, NCDs are universal but care and treatment facilities are not. Hence, still an ounce of prevention is worth a pound of cure.

Technology and research are rapidly growing, and deeper insights into diseases and mechanisms are paving the way for better treatments in the near future. Healthcare is an important benchmark of a country's progress and hospitals are the tangible indicators of the healthcare. More so, day by day health care is becoming digital which assists more in real time analysis and monitoring, prevention and patient empowerment. Digital medicine is the need of the era and can be delivered on a broad scale at low cost with the use of smartphones. It will help in treatment adherence and taking better care of the self, through personalized intervention overseen by the patients' own physicians, remote monitoring and follow up, and behavior modification which all ultimately improve outcomes in such costly and tough-to-manage NCDs. However, a solid personalized individual treatment plan and a robust health system to care for the patient must be in place for success of the digital medicine. Therefore, health care is also in transformation stage which is more preventive in nature, technology enabled, and patient centric. In fact, whole health ecosystem is changing. Hence, we also need to think differently and modify the measuring indicators for NCDs like.

1. No. of Yoga / Gym centres / 1,000 population

2. Pizza / Burger shops / 1,000 population

3. Walking track / 1,000 population

4. Cars - four wheelers / 1,000 population

5. Bicycles / 1,000 population

6. Medical Insurance coverage

7. No. of patient support groups / 1,000 patient population (any Chronic disease)

8. Happy health hours for terminally ill patients

There are 2 types of persons, ${ }^{1}$ healthy (apparently healthy) - the goal should be keep them healthy and ${ }^{2}$ disease /ill persons - the goal should be curative care and preventing the complications. However, the irony is that we take better care of our smart phones than ourselves. NCDs interventions can be designed based on available local evidences like. Patel et al reported that obesity, family history of diabetes, dyslipidaemia, uncontrolled glycaemic status, sedentary lifestyles, and hypertension were prevalent among the T2DM population. ${ }^{3}$ Independent predictors for diabetic diet consumption among T2DM population were visit to dietician, level of education, intake of low fat, and family history of diabetes. ${ }^{4}$ The characterization of these type of risk profile may contribute to designing more effective and specific strategies for screening and controlling diabetes.

\section{Possible interventions at individual level}

Create Awareness and Health promotion which includes...

1. Educate yourself

2. Check blood pressure and blood sugar regularly

3. Avoid tobacco and alcohol

4. Avoid drinking too much sweetened drinks

5. Low fat and low salt diet

6. Consume lot of green vegetables and fruits (Eat banana daily - avoid if diabetes is present) 
7. Avoid stress as possible

8. Walking / exercise daily for 20 minutes,

9. Regular Screening with some basic tests

\section{Possible Interventions at Population Level:}

1. Local adaptation and proper implementation of national program for prevention and control of cancer, Diabetes, cardiovascular diseases and stroke (NPCDCS)

Few NCDs strategic approaches for proper implementation of NPCDCS program are:

i. Formation of a high level technical advisory group

ii. Establishing partnership with State Health Department and National Health Mission

iii. Developing implementation guidelines/operational manual

iv. Skill development of frontline health workers (FLHWs)

v. Community Empowerment: Empowering the community with knowledge regarding NCDs through BCC (Behaviour change and communication) approach

vi. Involvement of local CBOs, CSOs which assists the local health system to develop a continuum of care approach for addressing the NCDs problem effectively-

a. Assisting health system in the screening of targeted population for NCDs

b. Linking the diagnosed patient to the treatment at health care facility

c. Follow up of at risk population through a robust follow up mechanism for lifestyle changes

d. Linking the complicated cases of cancer, hypertension and diabetes to higher (tertiary) level of health facilities for their management

vii. Development of robust Management Information System (MIS) and monitoring and evaluation tools and framework for real time data and regular monitoring the activities

2. Robust follow up mechanism for treatment adherence. M-health and E-clinic may work for reaching to the unreached population

3. Capacity strengthening at all levels (from top to bottom)

4. Management of the NCDs should be individual patient specific rather than general management of the condition

5. Separate cadre of healthcare workers especially for meticulous follow up

6. Family specific awareness generation

7. Use of very basic level screening and diagnostic tests

8. Policy level decision: NCDs should not be viewed and treated like communicable diseases.

9. Involvement of family members

10. Regular/weekly interactions among patients (Patient support groups)
We have solutions in hands, however it just requires implementation, implementation and implementation of NPCDCS program with more focus on restraining tobacco and alcohol use, unhealthy diets and promotion of physical activity.

India should be known for reducing the NCDs burden through 5-S approach; less consumption of salt and sugar, life(s)tyle modification, self-care and use of software. This really would help our nation from crippling problem of NCDs, which are taking a toll on the young and old alike. This requires collective wisdom, intra and inter-sectoral convergence and crosslearning. Civil society organisations (CSOs) and community based organisations (CBOs) can help Government in implementing some of the strategies at their level. As regards the importance of timings to an initiative to combat NCDs, it was noted, we can pay now or we can pay later. The cost will only rise with delay.

\section{References}

1. Upadhyay RP. An Overview of the Burden of NonCommunicable Diseases in India. Iranian J Publ Health 2012, 41 (3):1-8. https://www.ncbi.nlm.nih.gov/pmc/articles/PMC3481705

2. Beaglehole R, Bonita R, Kjellstrom T. Epidemiology and prevention. In, Basic Epidemiology, WHO Library Catalogue, 1993, 84.

3. Patel M, Patel IM, Patel YM, Rathi SK. A Hospital-based Observational Study of Type 2 Diabetic Subjects from Gujarat, India. J Health Popul Nutr. 2011;29(3):265-72. http://www.ncbi.nlm.nih.gov/pubmed/21766562

4. Patel M, Patel IM, Patel YM, Rathi SK. Factors associated with consumption of diabetic diet among Type 2 Diabetic Subjects from Ahmedabad, Western India. $J$ Health Popul Nutr. 2012;309(4):447-455. http://www.ncbi.nlm.nih.gov/pubmed/23304911 